\title{
Ultrafast dynamics in helium nanodroplets probed by femtosecond time-resolved EUV photoelectron imaging
}

\author{
Oleg Kornilov ${ }^{1,2}$, Chia C. Wang ${ }^{1,2+}$, Oliver Bünermann ${ }^{1,2}$, Andrew T. Healy ${ }^{1 \mathrm{x}}$, \\ Mathew Leonard ${ }^{1,2}$, Chunte Peng ${ }^{1,2 \dagger}$, Stephen R. Leone ${ }^{1,2 \bullet}$, Daniel M. Neumark ${ }^{1,2}$, \\ and Oliver Gessner ${ }^{1}$ * \\ ${ }^{1}$ Ultrafast X-ray Science Laboratory, Chemical Sciences Division, Lawrence Berkeley \\ National Laboratory, Berkeley, CA 94720 \\ ${ }^{2}$ Department of Chemistry, University of California, Berkeley, CA 94720
}

\begin{abstract}
The dynamics of electronically excited helium nanodroplets are studied by femtosecond time-resolved photoelectron imaging. EUV excitation into a broad absorption band centered around $23.8 \mathrm{eV}$ leads to an indirect photoemission process that generates ultraslow photoelectrons. A $1.58 \mathrm{eV}$ probe pulse transiently depletes the indirect photoemission signal for pump-probe time delays $<200 \mathrm{fs}$ and enhances the signal beyond this delay. The depletion is due to suppression of the indirect ionization process by the probe photon, which generates a broad, isotropically emitted photoelectron band. Similar time scales in the decay of the high energy photoelectron signal and the enhancement of the indirect photoemission signal suggest an internal relaxation process that populates states in the range of a lower energy droplet absorption band located just below the droplet ionization potential (IP $23.0 \mathrm{eV}$ ). A nearly $70 \%$ enhancement of the

Current addresses: ${ }^{+}$Department of Chemistry, University of British Columbia, Vancouver, British Columbia, Canada V6T 1Z1, ${ }^{\mathrm{x}}$ Department of Chemistry, University of Minnesota, Minneapolis, MN 55455, ${ }^{\dagger}$ Department of Chemistry, Massachusetts Institute of Technology, Cambridge, MA 02139

- On appointment as a Miller Research Professor in the Miller Institute for Basic Research in Science.

"Corresponding author. Email: ogessner@lbl.gov
\end{abstract}


ultraslow photoelectron signal indicates that interband relaxation plays a more dominant role for the droplet de-excitation mechanism than photoemission.

\section{Introduction}

Since the early 1990's, helium nanodroplets $\mathrm{He}_{\mathrm{N}}, \mathrm{N}>10^{3}$, have attracted a steadily increasing amount of attention across a number of scientific communities. ${ }^{1-3}$ Today, clusters of ${ }^{4} \mathrm{He}$ atoms are probably best known for their use as a superfluid host that enables spectroscopic studies on regular and exotic species at temperatures of $\sim 0.4 \mathrm{~K} .^{4-12}$ He droplets exhibit a unique combination of cryogenic temperatures, superfluidity, nanoscale dimensions, excellent dopant pickup capability, high effective heat capacity, and high electronic excitation energies. These properties continue to inspire a range of proven and proposed applications, from providing nano-scale reaction chambers ${ }^{13,14}$ to hosting biological samples in ultrafast single shot diffractive imaging experiments at 4 th Generation Light Sources. ${ }^{15-17}$

In this work, we investigate the femtosecond time-resolved electronic and nuclear dynamics of pure large $\left(\mathrm{N} \sim 2 * 10^{6}\right)$ helium droplets following extreme ultraviolet (EUV) excitation into a broad absorption band spanning the photon energy range from the droplet ionization potential (IP) at $\sim 23.0 \mathrm{eV}$ to the atomic helium IP at $24.6 \mathrm{eV}$. Fluorescence excitation measurements by Möller and co-workers ${ }^{18}$ have shown that photoexcitation of helium droplets in this energy range leads to the emission of atomic helium Rydberg states and highly ro-vibronically excited helium molecules.

Photoionization experiments by Fröchtenicht and co-workers ${ }^{19}$ and photoelectron imaging studies by Peterka and co-workers ${ }^{20,21}$ have demonstrated that photoionization 
within this range proceeds via an indirect process. Most remarkably, the photoelectron images are largely independent of photon energy and comprise a single feature with an intensity maximum close to zero and a full-width-at-half-maximum (FWHM) of less than $1 \mathrm{meV}$.

Thus far, it has been challenging to identify the mechanism by which these slow electrons are produced. Several interpretations have been proposed to explain this result as well as other evidence for electron energy loss following indirect ionization of pure and doped He droplets. ${ }^{12,18-20,22-26}$ For example, energy loss may result from the formation of cavities inside the droplet, so-called "bubbles", that enclose defects such as photoelectrons or Rydberg atoms, efficiently transferring energy to the bulk during their formation and the excited regions' travel to the droplet surface where the bubbles' contents is released with very small kinetic energy. ${ }^{20-22,27-30}$ It is also possible that slow electrons are formed by vibrational autoionization of Rydberg states, i.e. the transfer of vibrational to electronic energy facilitated by neutral states that are embedded in the ionic continuum..$^{20,21}$

With the recent advent of femtosecond pulse duration EUV light sources ${ }^{31-34}$ an entirely new set of tools becomes available to probe the dynamics of helium droplets after electronic excitation. Here, we present the first femtosecond time-resolved study of the electronic and nuclear dynamics in helium droplets, probing the electronic states prepared in the EUV that lead to the emission of photoelectrons below the atomic ionization threshold and to the emission of neutral helium Rydberg atoms and molecules. The principle of the experiment is outlined in Fig. 1. Droplets are excited by a femtosecond pump pulse with a photon energy centered around $\sim 23.7 \mathrm{eV}$. The evolution of 
electronically excited states is monitored by subsequent ionization using a $1.58 \mathrm{eV}$ (785 $\mathrm{nm}$ ) probe pulse that is delayed by up to 100 ps relative to the pump pulse.

With this setup, both the droplet EUV photoionization mechanism as well as the desorption of helium Rydberg atoms and molecules can be probed with femtosecond time-resolution. For example, most He* $(1 \mathrm{~s} n l)$ Rydberg states with $n>2$ can be ionized by

the probe pulse. These states have very distinct photoelectron images ${ }^{35}$ which permits direct probing of their rates of production. In addition, the probe pulse should deplete the population of the electronically excited droplets created by the EUV pump pulse, resulting in depletion of the low energy electron signal at early times and recovery at later times. The presented data indeed show evidence for these and other effects, yielding new insights into the decay mechanisms of electronically excited droplets. Specifically, a depletion of the low energy photoelectron signal at pump-probe time delays below $\sim 200 \mathrm{fs}$ is observed, followed by an enhancement at longer delays. The findings are interpreted in terms of a relaxation mechanism in which the droplet undergoes a transition from an initially excited electronic band to an energetically lower-lying band from which it can be re-excited into the upper band by the probe pulse. This picture is supported by the observation of a broad photoelectron band that decays on a time scale that is comparable to the rise time of the low energy photoelectron enhancement.

\section{Experiment}

The experiments are carried out using a new apparatus designed for femtosecond time-resolved photoelectron imaging studies in the EUV (Fig. 2). A femtosecond EUV light source based on high-order harmonic generation is interfaced to a previously 
designed He droplet instrument with photoelectron imaging capabilities. ${ }^{36}$ Femtosecond duration EUV pulses are generated in the nonlinear interaction of an intense femtosecond infrared (IR) laser pulse with a gaseous medium. ${ }^{37-40}$ The co-propagating fundamental IR light is removed from the EUV beam path by a two-stage silicon-based dichroic mirror ${ }^{40}$ followed by a $100 \mathrm{~nm}$ thick aluminum filter. In a second beam branch, a small fraction of the IR laser light is routed through a retro-reflector mounted on a computer-controlled translation stage allowing for the adjustment of the relative time delay between the two branches. The IR beam is recombined with the EUV beam by means of an annular mirror. The EUV beam passes through the central hole while the IR beam is reflected off the outer parts of the mirror. A curved multilayer mirror focuses both beams on the gas target. The layer structure of a $\mathrm{Mg} / \mathrm{SiC}$ mirror is optimized to reflect at $\mathrm{h} v=23.7 \mathrm{eV}$ (15th harmonic) and to suppress other EUV components. A high specular IR reflectivity of the mirror allows to simultaneously focus both beams with a single optical component. The position of a concave lens in the IR beam path is optimized to achieve the same effective focal length for both EUV and IR beams enabling maximum overlap of the two focal volumes.

The commercial femtosecond IR laser system (Kapteyn-Murnane Laboratories Red Dragon) provides pulses with a center wavelength of $785 \mathrm{~nm}$, a duration of $25 \mathrm{fs}$ (FWHM) and energies of up to $5 \mathrm{~mJ}$ at a $3 \mathrm{kHz}$ repetition rate. Pulse energies of 2.5-3 mJ are used for the experiments described herein. The fundamental IR beam is focused into a gas cell by a concave mirror with a $200 \mathrm{~cm}$ nominal focal length. The cell consists of a stainless steel tube that is sealed by $50 \mu \mathrm{m}$ thick nickel foils on both ends. The IR beam drills pinholes into the foils that transmit the laser and the harmonic light. The distance 
between the nickel foils defines a cell length of $2.5 \mathrm{~cm}$. The cell is filled with krypton or xenon at a typical pressure of $\sim 4$ Torr $(\sim 530 \mathrm{~Pa})$, which is maintained by a closed-loop controlled needle valve. The achieved relative pressure stability of better than $0.1 \%$ has proven to be instrumental for the overall reproducibility of the experiment. The fundamental IR beam enters and exits the gas cell through holes in the nickel foils that are drilled by the laser beam itself within a fraction of a second. The resulting gas flow out of the cell leads to a background pressure of about $10^{-4}$ Torr in the surrounding vacuum chamber, which is reduced to less than $10^{-8}$ Torr downstream by differential pumping along the EUV propagation direction.

The dichroic mirror consists of two blocks of silicon which are positioned such that their superpolished surfaces are aligned parallel with respect to each other and at a $75^{\circ}$ incidence angle with respect to the IR beam. This Brewster's angle geometry leads to an efficient absorption of the IR beam (extinction $\sim 10^{4}$ ) while the harmonic radiation is reflected with an average reflectivity of $\sim 40 \%$ on each silicon surface. ${ }^{40}$ Further suppression of residual IR contributions in the EUV beam is provided by a $100 \mathrm{~nm}$ thick aluminum filter, which has $\sim 80 \%$ transmission in the EUV region of interest. ${ }^{41}$ The spectrum and spatial mode of the EUV beam is analyzed by means of a homebuilt in situ EUV spectrometer/beam-profiler described in detail elsewhere. ${ }^{42}$ An example for an EUV spectrum is shown in the inset of Fig. 2.

Selection of the desired harmonic is performed with a $\mathrm{Mg} / \mathrm{SiC}$ multilayer mirror that has a nominal focal length of $50 \mathrm{~cm}$ and is provided by the Center for X-ray Optics (CXRO) at Lawrence Berkeley National Laboratory. The multilayer mirror reflectivity in the optimized photon energy range of the 15 th harmonic is determined to $\sim 30 \%$. We note 
that other harmonic orders are reflected with non-negligible efficiency. However, their contribution to the results is deemed to be minor due to their large energy spacing and negligible overlap with any structure in the helium droplet absorption spectrum. The total EUV photon flux (pump pulse) in the interaction volume is determined to $\sim 5 \times 10^{6}$ photons/pulse ( $20 \mathrm{pJ} /$ pulse) using a calibrated VUV photodiode (International Radiation Detectors AXUV100G). The multilayer mirror also focuses the IR light from the second branch ( $\sim 5 \mu \mathrm{J}$ of pulse energy), used as the probe pulse, into the interaction volume.

The helium droplet apparatus and photoelectron imaging setup are described in detail elsewhere. ${ }^{36}$ Briefly, helium droplets are generated by expanding helium gas (Airgas, $99.9999 \%$ purity) at temperatures of $10-20 \mathrm{~K}$ and pressures of $20-80$ bar through a $5 \mu \mathrm{m}$ orifice into a vacuum chamber. After expansion and droplet formation, the beam passes through two skimmers with $2 \mathrm{~mm}$ orifice diameters before entering the interaction volume. The source pressure and temperature in the current experiments are set to $80 \mathrm{bar}$ and $13 \mathrm{~K}$, respectively. Under these conditions, the average droplet size is expected to be $\mathrm{N} \sim 2 * 10^{6}$ atoms. ${ }^{1}$ Images of free helium atoms are recorded either by introducing an effusive helium beam into the interaction volume from a nearby gas cell or by operating the droplet source in a regime of temperatures and pressures that inhibit the formation of any helium clusters.

Photoelectrons are imaged onto a microchannel plate-based detector by means of a Velocity-Map-Imaging (VMI) spectrometer. ${ }^{43}$ The back of the detector is equipped with a phosphor screen producing photoelectron images in the visible that are recorded with a high-resolution high-sensitivity CCD camera. The electric polarization vectors of both 
linearly polarized beams are aligned parallel with respect to each other and perpendicular with respect to the extraction direction of the photoelectrons. Under these conditions, the VMI setup allows to extract both photoelectron kinetic energies and photoelectron angular distributions (PADs) from the 2D images.

For each pump-probe time delay, two images are recorded: one with both beams present and a second using the EUV beam only. By analyzing the difference of the two images, most signal background contributions due to parasitic harmonic light and camera noise can be readily removed. The total photoelectron count rate is estimated to be about $1 \mathrm{kHz}$ or less, requiring acquisition times in the range from $10 \mathrm{~s}$ to $1 \mathrm{~min}$ per image. The time delays are varied in steps of 20 fs to 2 ps depending on the total time delay range of the data set. For each data set, several sweeps across the entire range of time delays are recorded with alternating sweep directions to minimize the impact of any systematic signal variations during the course of the experiment on the measured transient photoelectron images. From the measured rise time of the atomic helium photoelectron signal corresponding to ionization of transiently populated Rydberg states, the apparatus function (pump-probe cross-correlation function width) is found to be $100 \mathrm{fs}$ (FWHM).

\section{Results}

Complementary signals from direct and indirect photoionization processes dominate different parts of the transient photoelectron images. Indirect droplet ionization below the atomic IP leads to an intense photoelectron signal that is restricted to a very small region in the center of the detector. In contrast, direct ionization by the probe pulse promotes photoelectrons to higher kinetic energies and thus towards the outer area of the 
detector. The experimental challenge is to disentangle the transient signal contributions in both detector regions and to combine the results in a unified picture.

\section{A. Transient photoelectron images and spectra}

Fig. 3 shows the typical evolution of photoelectron images as a function of pumpprobe time delays for droplets $\left(\mathrm{N} \sim 2 * 10^{6}\right.$, upper part) and free atoms (lower part). Note that for time delays $\geq 0 \mathrm{fs}$, the sequence of images shows the difference between the "pump+probe" and "pump only" signals. The atomic PADs and energy distributions in Fig. 3 are readily described by IR-photoionization of transiently populated atomic Rydberg states. This process has recently been discussed in depth by Haber et al. for selected $1 \mathrm{~s} 3 \mathrm{p}$ and $1 \mathrm{~s} 4 \mathrm{p}$ states. ${ }^{35}$ In brief, the three structures correspond, in increasing distance from the center, to ionization of atomic $1 \mathrm{~s} 3 \mathrm{p}\left({ }^{1} \mathrm{P}\right), 1 \mathrm{~s} 4 \mathrm{p}\left({ }^{1} \mathrm{P}\right)$, and $1 \mathrm{~s} 5 \mathrm{p}\left({ }^{1} \mathrm{P}\right)$ Rydberg states that are excited within the energy bandwidth of the pump pulse. The corresponding signals appear stationary within the range of positive pump-probe delays presented here due to the long lifetimes which are on the order of $1.7 \mathrm{~ns}, 3.9 \mathrm{~ns}$ and $7.7 \mathrm{~ns}$

for the $3 p, 4 p$ and $5 p$ excitations, respectively. ${ }^{35,44,45}$

In contrast, the photoelectron images of helium droplets show significantly different features and trends. At negative pump-probe delay times (IR pulse precedes EUV pulse) only one strong peak is observed at the center of the detector. This feature corresponds to the one-photon EUV photoelectron spectrum that has previously been observed in synchrotron-based photoionization studies ${ }^{20}$ with dominant contributions below $\sim 1 \mathrm{meV}$ kinetic energy. Droplets that have not been excited by an EUV photon are 
transparent to the IR probe beam. ${ }^{1}$ Therefore, no effect of the IR beam on the droplet photoelectron images is observed for negative pump-probe delay times.

The images change dramatically as soon as the pump- and probe-beams overlap in time, and time-dependent changes in the photoelectron images are readily discernible over hundreds of femtoseconds for positive pump-probe delays. A broad ring covering a detector area similar to the atomic Rydberg 1s4p and 1s5p photoionization peaks dominates the images at time delays below $\sim 250$ fs. The intensity of the ring decays rapidly with increasing time delay and becomes weak compared to the sharply structured features that correspond to photoemission along the light polarization vector. These features have a striking resemblance to the atomic helium photoelectron images and dominate the droplet spectra for time delays beyond $\sim 500 \mathrm{fs}$. The signal intensity in the image center region exhibits transient changes, which are complementary to those of the signal in the outer detector regions. Depletion of the center signal at time-delays $\leq 250 \mathrm{fs}$ is followed by a significant enhancement at longer delays that approaches its asymptotic limit on a picosecond time scale.

In order to maximize the contrast between dynamics of different image contributions in the subsequent analysis, different angular emission angle ranges will be distinguished as sketched in Fig. 3. The "parallel range" (PR) includes all emission angles within $\pm 40^{\circ}$ cones around the light polarization vector. The "sideways range" (SR) includes all emission angles within ranges $54^{\circ}<\theta<70^{\circ}$ and $-70^{\circ}<\theta<-54^{\circ}$ relative to the light polarization vector. The ranges have been chosen such that they include maximum (PR) and minimum (SR) contributions from purely atomic PADs, respectively. To guide the eye, an ideal d-wave PAD (angular momentum and projection quantum numbers $1=2$, 
$\mathrm{m}=0$ ) has been included in Fig. 3 . From the work of Haber et al. ${ }^{35}$ it is known that this is the dominant PAD contribution from atomic helium, in agreement with the measurements presented here.

The 2D false color plots in Figs. 4a,b show the transient photoelectron kinetic energy spectra for PR and SR angular emission directions. The spectra were derived by applying an inverse Abel transform to the raw photoelectron images employing a socalled BASEX basis function set. ${ }^{46}$ This inversion procedure results in $2 \mathrm{D}$ images in which the radial coordinate (distance from the image center) corresponds to the electron momentum and the angular distribution in the image plane directly corresponds to the PAD in 3D space. Figs. 4a,b are the result of an integration of the inverted images over the two different angular emission ranges indicated in Fig. 3. The spectra in Figs. 4d,e,f on the right hand side of Fig. 4 are generated by integrating the 2D intensity distributions on the left hand side over pump-probe time delays $0 \mathrm{fs} \leq \Delta \mathrm{t} \leq 500 \mathrm{fs}$.

The ring-shaped structure, which is characteristic for the raw droplet images in Fig. 3, appears as a broad horizontal band at early pump-probe delay times in Figs. 4 a,b. At time delays beyond $\sim 2$ ps, the electron momentum distribution in Fig. 4a is clearly dominated by two well-defined structures. In contrast, Fig. $4 \mathrm{~b}$ shows only very weak signal at these longer times. The distinction becomes even more evident in Fig. 4c, which shows the difference of Figs. $4 \mathrm{a}$ and $4 \mathrm{~b}$, normalized to the same integration area on the detector. The spectral structure of the difference is very similar to an atomic helium spectrum (Fig. 4f). The indicated peak positions correspond to photoionization of atomic Rydberg states with electron configurations $1 \mathrm{~s} 4 \mathrm{p}\left({ }^{1} \mathrm{P}\right)$, and $1 \mathrm{~s} 5 \mathrm{p}\left({ }^{1} \mathrm{P}\right)$, respectively, by an IR probe photon. 


\section{B. Analysis}

In order to perform a quantitative analysis of the timescales and relative contributions of different spectral features, the spectral intensities of Figs. 4a-c are integrated over momentum ranges covered by the photoelectron peaks marked with $4 p$ and 5p. The results are shown in Figs. 5a-c. The transient signals of PR and SR electrons both comprise a fast ( $280 \mathrm{fs}$ ) decaying component and a component that decays on a considerably slower timescale ( $\sim 2.8 \mathrm{ps})$. Remarkably, the difference between the curves (after normalization to identical detector integration areas, Fig. 5c) does not show any of the decay that is very prominent in the PR and SR components. Consequently, the decaying components have similar intensities per unit emission angle for both parallel and sideways emission angles. Given the geometry of the setup, including the parallel alignment of pump- and probe-beam polarization vectors, we conclude that the decaying components correspond to an isotropic PAD with a broad kinetic energy distribution as shown in Figs. 4b,e.

The solid curves in Fig. 5 are the result of a fit to a model function that represents the sum of three transient contributions: two single-exponential decays with decay time constants of $\tau_{1}=280$ fs and $\tau_{2}=2.8 \mathrm{ps}$ and a step function which is zero at negative time delays and has a constant value $\mathrm{A}_{3}>0$ at positive time delays. The use of a step function as the third component that describes the asymptotic behavior of the PR and SR transients was derived from Fig. 5c. It shows that the difference between the signals in parallel and sideways emission directions can be well described by a function that rises instantly to a constant value within our experimental resolution. All contributions have been 
convoluted with the apparatus function during the fit procedure. The different components of the fit function are indicated as dashed lines in Fig. 5. By performing fits to the data using the same model function and a variety of different but fixed time scales, the true timescales are estimated to be within the limits of $\tau_{1}=280 \mathrm{fs}+100 \mathrm{fs} /-50 \mathrm{fs}$ and $\tau_{2}=2.8 \mathrm{ps} \pm 0.4 \mathrm{ps}$.

We note that due to a mathematical ambiguity, identical fit qualities can by achieved by describing the decay behavior by either a parallel or a sequential model. In the parallel model, two single exponential decay functions with independent time constants are employed, corresponding to the physical model of two independent decay processes that proceed on different time scales. Before convolution with the apparatus function, the fit function is:

$$
I(\Delta t>0)=A_{1} \exp \left(-\frac{\Delta t}{\tau_{1}}\right)+A_{2} \exp \left(-\frac{\Delta t}{\tau_{2}}\right)+A_{3}
$$

The amplitudes $A_{1,2,3}$ and the time constants $\tau_{1,2}$ are free fit parameters. In the sequential model, a single exponential decay described by $\tau_{1}$ and a double exponential decay containing both $\tau_{1}$ and $\tau_{2}$ are employed. In this model, the two decay processes are correlated such that the decay of an initially excited state within $\tau_{1}$ feeds population into an intermediate state, which decays within a time scale $\tau_{2}$ :

$$
I(\Delta t>0)=A_{1} \exp \left(-\frac{\Delta t}{\tau_{1}}\right)+A_{2}\left[\exp \left(-\frac{\Delta t}{\tau_{2}}\right)-\exp \left(-\frac{\Delta t}{\tau_{1}}\right)\right]+A_{3}
$$

Provided $\mathrm{A}_{1}>0$, equations (1) and (2) lead to identical fit qualities, the fit results only differ in the magnitudes of $\mathrm{A}_{1}$ and $\mathrm{A}_{2}$.

Of particular interest for the droplet photoionization dynamics is the transient intensity of the photoelectron signal at the center of the detector. This is the only signal 
observed for one-photon EUV photoionization below the IP of atomic helium. ${ }^{20}$ Fig. 6a shows the ratio of the pump-probe signal and the signal that is observed for excitation with the EUV pump pulse only: $\mathrm{I}_{\text {center }}(\Delta \mathrm{t})=\mathrm{I}($ pump + probe, $\Delta \mathrm{t}) / \mathrm{I}($ pump$)$. The decrease in intensity just after zero pump-probe delay corresponds to the anticipated depletion of the ultraslow photoelectron signal. The observation of values $\mathrm{I}_{\mathrm{c}}(\Delta \mathrm{t})>1$ for $\Delta \mathrm{t}>200 \mathrm{fs}$, however, is entirely unexpected. Fig. 6a shows that the rise of $I_{c}$ to greater positive time delays indeed continues over an extended period of time and can be described by the sum of two exponentially rising contributions, characterized by the time constants $\tau_{4}=140 \mathrm{fs} \pm 40 \mathrm{fs}$ and $\tau_{5}=2.5 \mathrm{ps}+0.4 \mathrm{ps} /-0.2 \mathrm{ps}$. Note that $\tau_{5}$ is very similar to the time constant that describes the slowly decaying component in Fig. 5. Remarkably, the photoelectron spectrum associated with the additional amount of slow electrons in the pump-probe experiment is indistinguishable from the photoelectron spectrum generated by the EUV pump pulse alone (Fig. 6b).

In summary, the main results of this analysis can be categorized into three spectral signatures of droplet relaxation dynamics: 1) an isotropically emitted, energetically broad photoemission band exhibiting dynamics that are predominantly described by the sum of a fast ( $\sim 280$ fs) and a slowly ( $2.8 \mathrm{ps})$ decaying component. 2$)$ An instantly rising photoelectron signal characterized by a kinetic energy spectrum corresponding to production of atomic $1 \mathrm{~s} 4 \mathrm{p}$ and $1 \mathrm{~s} 5 \mathrm{p}$ states, and an angular distribution that is similar to that of isolated helium atoms. 3) A very low energy photoelectron component that shows a complex transient behavior. Depletion of the signal at small pump-probe time delays $(<200 \mathrm{fs})$ is followed by an overshoot of the signal compared to negative time delays. The signal continues to rise for longer time delays and can be well described by 
contributions from two exponentially rising components with time constants of $\tau_{4}=140$ fs \pm 40 fs and $\tau_{5}=2.5 \mathrm{ps}+0.4 \mathrm{ps} /-0.2 \mathrm{ps}$.

\section{Discussion}

\section{A. Relaxation from the upper to the lower electronic band}

Excitation of the droplet in the absorption band centered around $23.8 \mathrm{eV}$ populates states that encompass a sufficiently large number of atoms to form a continuous electronic band. The decay of this initially excited band is marked by two significantly different time scales, $280 \mathrm{fs}$ and $2.8 \mathrm{ps}$. The longer time constant also describes, within the error of the measurements, the long-term rise of the ultraslow electron peak beyond the intensity that is induced by a single EUV photon excitation of the droplet. This result implies that the slow decay of the excited state populates a state that, when excited by the IR probe pulse, produces very slow electrons whose kinetic energy spectrum is indistinguishable from those produced by the EUV pulse alone (Fig. 6b).

A mechanism consistent with these observations in shown in Fig. 8, in which we suggest that the excitation state around $23.8 \mathrm{eV}$ undergoes a radiationless transition to a lower-lying excited state of the droplet. This lower state then absorbs a probe photon to re-populate the initially excited state, which can then decay by slow electron emission just as in the EUV-only experiment. As shown in the left panel of Fig. 8, there is a lowerlying droplet excited state responsible for a broad absorption band centered at $21.6 \mathrm{eV}^{47}$ that is in the right energy range for this mechanism to be feasible. The probe-pulse induced enhancement of the ultraslow photoelectron signal amounts to almost $70 \%$ of the 
single EUV photon induced intensity. This suggests that relaxation from the upper into the lower electronic band is fast compared to the emission process of the ultraslow photoelectrons. In other words, photoemission is a minor droplet relaxation mechanism compared to interband relaxation.

\section{B. Parallel vs. sequential relaxation mechanisms}

While the slower 2.8 ps time scale for relaxation from the upper electron band is reflected in the long-term rise of the ultraslow photoelectron signal, no rising component is detected that would complement the short 280 fs decay time constant. One possible scenario that is compatible with this observation is that the short and the long time constant are associated with two independent physical processes that both commence instantaneously after EUV excitation of the droplet but then progress on different time scales (Eqn. 1, Fig. 8a) and lead to different products. In this scenario, only the slower process leads to the population of the lower electron band and the corresponding additional intensity in the slow electron peak while the faster process does not. The short time scale could, for example, be associated with the emission of excited helium fragments that have been detected by Möller and co-workers. ${ }^{18}$ Upon emission of excited helium atoms and molecules, most of the electronic excitation energy would be transferred to the emitted fragments, leaving the droplet in a state that cannot be reexcited to energies above the IP by the IR probe pulse. This would lead to the observed absence of the $280 \mathrm{fs}$ time scale in the slow electron signal overshoot. In contrast, excited $\mathrm{He}^{*}(1 \mathrm{~s} n l, n>2)$ atoms that are emitted by the droplet can be ionized by the probe pulse and would lead to long-lived spectral features similar to those detected in pump-probe 
experiments on isolated $\mathrm{He}$ atoms, as has been observed in the droplet measurements (Figs. 3-5). However, no evidence for a 280 fs rise time of the long-lived spectral components has been observed; instead these components seem to emerge within the time resolution of the experiment ( 100fs, Fig. 5c). Consequently, the $280 \mathrm{fs}$ decay would have to result in the population of a possible dark state that cannot be ionized with the IR probe pulse. Furthermore, the parallel relaxation model requires the initial excitation of two independent states within the upper electronic band that have very similar photoelectron spectra and PADs but that decay on time scales that differ by an order of magnitude. This might be possible but requires the additional assumption that two states with similar electronic characters within the droplet are decaying via significantly different mechanisms. To conclude, the physical picture that emerges from the implementation of the parallel model is rather complex and requires the existence of a dark state that is populated by the $280 \mathrm{fs}$ decay and that cannot be ionized by the probe pulse.

An alternative droplet relaxation mechanism is sketched in Fig. 8b. In this case, initial droplet excitation is followed by a population transfer within the upper electronic band proceeding on a time scale of $\sim 280 \mathrm{fs}$. This intraband transition is followed by relaxation to the lower electronic band on a time scale of $\sim 2.8$ ps (Eqn. 2). This sequential model naturally explains the absence of a 280 fs rising component in the slow electron transient since the model function that describes the product growth from a sequential relaxation mechanism comprised of an initial fast $\left(\tau_{1}\right)$ and a slow $\left(\tau_{2}\right)$ decay process does not contain a rising slope that directly reproduces the shorter time scale: 


$$
I(\Delta t>0)=A_{4}\left[1-\frac{1}{1-\frac{\tau_{1}}{\tau_{2}}} \exp \left(-\frac{\Delta t}{\tau_{2}}\right)-\frac{1}{1-\frac{\tau_{2}}{\tau_{1}}} \exp \left(-\frac{\Delta t}{\tau_{1}}\right)\right]
$$

Note that for $\tau_{1}<<\tau_{2}$, this expression does not differ significantly from a single exponential rise with time constant $\tau_{2}$. Within the experimental errors, the description of the ps-range slope of the data presented in Fig. 6a by Eqn. 3 leads to the same fit quality as a description by a single exponential rise.

The parallel and the sequential models yield the same fit qualities but their physical interpretations differ in complexity. The sequential model is more appealing since it requires a less complex physical description and no hypothesized dark states in order to explain the lack of a 280 fs rise time in any of the photoelectron signals. In particular the absence of dark states in the sequential model is a significant advantage of this mechanism since one of the strengths of a photoionization experiment is that there are in principle no dark states within the limits of energy conservation.

A more detailed picture of the microscopic physical processes that are associated with the intraband and interband relaxations would be desirable. Theoretical and experimental studies of Apkarian, Janda, Halberstadt and co-workers ${ }^{48-50}$ on small He clusters and bulk liquid helium indicate that electron hole delocalization and dimerization to form $\mathrm{He}_{2}{ }^{+}$after removal of an electron proceed on time scales on the order of several $100 \mathrm{fs}$ or less. Apkarian et al. ${ }^{51}$ observed a 3.5 ps decay time constant in femtosecond time-resolved laser induced fluorescence (LIF) studies on triplet state excimers in liquid helium. The decay was interpreted as the relaxation time of a bubble around the excited He dimer to accommodate a change in the electronic density distribution upon excitation from the $\mathrm{a}(2 \mathrm{~s})$ to the $\mathrm{c}(3 \mathrm{p})$ state. Theoretical studies of Eloranta, Apkarian, Rosenblit, and 
Jortner predict electron bubble relaxation time scales between $\sim 9$ ps and $\sim 130$ ps. $^{52,53}$ While it seems tempting to associate some of these timescales with the dynamics observed in our measurements, analogies between the different studies have to be drawn with great care. More experimental and, in particular, theoretical studies will be needed to disentangle the fundamental processes that underlie the cyclic excitation-relaxation scheme that has been presented in this work.

\section{Indirect ionization mechanism}

The details of the indirect ionization process remain elusive. The superposition of several signals in the ultraslow photoelectron peak voids the determination of a unique timescale for the indirect ionization mechanism. Yet, the magnitude of the ultraslow photoelectron signal overshoot towards long pump-probe time delays suggests that the indirect ionization mechanism is a minor process compared to relaxation from the upper to the lower electronic band of the droplet. In other words, photoemission proceeds at a lower rate than interband relaxation. We therefore conservatively use the interband relaxation rate as an upper bound for the photoemission rate and, equivalently, the interband relaxation time of $2.8 \mathrm{ps}$ as a lower bound for the duration of the indirect photoemission process. In both the parallel and the sequential model, photoemission can then be included as a minor product channel that is accessible as long as the droplet is in an excited state within the upper electronic band.

The origin of the initial $\sim 140$ fs rise of the slow photoelectron signal (Fig. 6a) is still to be determined. One possibility is that it corresponds to the photoelectron signal from an additional product channel such as excited $\mathrm{He}^{*}$ atoms in the triplet coupled 
$1 \mathrm{~s} 3 \mathrm{p}\left({ }^{3} \mathrm{P}\right)$ state. This product has been observed by Möller et al. ${ }^{18}$ in the relaxation of large helium clusters and its IP corresponds exactly to the center probe photon energy in our experiment which would explain its contribution to a very slow photoelectron signal. Other explanations for the 140 fs rise are conceivable but they require the use of more complex dynamical models such as a sequential process involving three consecutive transitions between a total of four states, which makes them less favorable.

\section{Atomic Rydberg states}

The energy and angular distributions of the sharp structures in the droplet photoelectron images (Figs. 3, 4f) and their association with long lived product states (Figs. 4c,f, 5c) suggest that these spectral contributions originate from isolated helium atoms. Theoretically, it cannot be excluded that this signal stems from a contamination of the droplet beam by isolated helium atoms or from background helium gas in the experimental chamber. However, from measured cluster size distributions ${ }^{54,55}$ we do not expect a significant contribution of atoms in the cluster beam. Furthermore, the PAD generated by the 17th harmonic leakage of the multilayer mirror is isotropic (not shown), which indicates that there is no prominent contribution of isolated helium atoms in the interaction volume that would otherwise produce an anisotropic PAD corresponding to an outgoing p-wave from direct He1s ionization.

Identifying excited droplets as the origin of the excited helium atoms suggests that the latter correspond to the He* atoms whose emission was first identified by the Möller group as a dominant droplet relaxation mechanism. ${ }^{18}$ The prompt rise of their spectral signature (Fig. 5c) indicates a very short emission time on the order of the experimental 
time resolution (100 fs). Within the precision of the experiment, this agrees with the 140 fs rise time of a possible Rydberg atom contribution to the slow electron signal discussed in the last section. We therefore tentatively propose a He* emission time scale on the order of $140 \mathrm{fs}$. This rather short timescale suggests that the He* atoms observed in this study are created at the droplet surface and are not transferred from the bulk to the surface by a more time-consuming bubble transport mechanism. ${ }^{27}$ We note that a time

scale of 180 fs has been observed by Stienkemeier and co-workers ${ }^{56}$ for the desorption of $\mathrm{K}^{*} \mathrm{He}$ exciplexes formed on the surface of helium droplets by electronic excitation of the potassium dopant. While this process is different from EUV excitation of the entire droplet, the results demonstrate that the repulsive interaction between neutral excited species and the droplet surface can generate atom and molecule ejection timescales in the range of those reported here. Our experiments do not exclude the existence of a bubble transport mechanism, but its investigation would require much larger pump-probe time delays than the ones used here.

\section{Conclusion}

Femtosecond time-resolved photoelectron images of electronically excited helium droplets with an average size of $\sim 2 \times 10^{6}$ atoms have been recorded. Emission of ultraslow photoelectrons after EUV excitation at $23.8 \mathrm{eV}$ is suppressed by the IR probe photon at pump-probe time delays of less than $200 \mathrm{fs}$ and enhanced for larger delays. This finding is explained by an interband relaxation mechanism that transfers population from the upper to the lower electronic band on a time scale of $2.8 \mathrm{ps} \pm 0.4 \mathrm{ps}$. This relaxation 
pathway is significantly more efficient than the photoemission process, yielding a conservative estimate of the lower bound for the photoemission time scale of $2.8 \mathrm{ps}$. A second relaxation mechanism is proceeding on a time scale of $280 \mathrm{fs}+100 \mathrm{fs} /-50 \mathrm{fs}$. It is yet to be determined if the two time scales are due to the existence of two fairly independent relaxation processes within the droplet, or if they are correlated by a sequential relaxation scheme in which a fast primary intraband decay is followed by a slower interband transition. The simplicity of the physical picture that is associated with the sequential model and the absence of any dark states in this description make it more appealing than the parallel model but further studies are needed to find hard evidence in favor of one or the other mechanism. A promptly rising photoelectron signal reminiscent of the spectroscopic footprint of isolated helium atoms indicates a rapid ejection of excited $\mathrm{He}^{*}$ fragments from the droplet. The short appearance time on the order of $140 \mathrm{fs}$ suggests that the atoms are ejected from the droplet surface and are not being transferred from the bulk to the surface in a more time-consuming bubble transport mechanism.

The wealth of new insights into the droplet relaxation dynamics that has been facilitated by the first time-domain study in the EUV is very encouraging. Nevertheless, it has also become evident that more experimental and in particular theoretical work is needed to uniquely identify the mechanism(s) that leads to the emission of electrons as well as Rydberg atoms and molecules after electronic excitation of pure helium droplets. Both efforts are under way: A combination of photoelectron imaging experiments with higher probe photon energies and transient ion imaging measurements will help to disentangle so-far ambiguous results such as the timescale of the droplet ionization process. Droplet size dependent studies will show to what extend the distinction between 
the surface and the bulk region of the nanodroplets plays a role in the relaxation dynamics. Ab-initio calculations on electronically excited droplet states and rovibronically excited fragments will provide an independent guidance for the interpretation of the experimental data.

\section{Acknowledgements}

This work was supported by the Director, Office of Science, Office of Basic Energy Sciences, Chemical Sciences Division of the U.S. Department of Energy under Contract no. DE-AC02-05CH11231. The authors thank the LBNL Center for X-ray Optics (CXRO) for providing custom-made multilayer mirrors.

\section{References}

(1) Toennies, J. P.; Vilesov, A. F. Angew. Chem. Int. Ed. 2004, 43, 2622.

(2) Choi, M. Y.; Douberly, G. E.; Falconer, T. M.; Lewis, W. K.; Lindsay, C. M.; Merritt, J. M.; Stiles, P. L.; Miller, R. E. Int. Rev. Phys. Chem. 2006, 25, 15.

(3) Stienkemeier, F.; Lehmann, K. K. J. Phys. B: At., Mol. Opt. Phys. 2006, 39, R127.

(4) Braun, A.; Drabbels, M. J. Chem. Phys. 2007, 127, 114303.

(5) Grebenev, S.; Toennies, J. P.; Vilesov, A. F. Science 1998, 279, 2083.

(6) Mudrich, M.; Stienkemeier, F.; Droppelmann, G.; Claas, P.; Schulz, C. P. Phys. Rev. Lett. 2008, 100, 023401.

(7) Denifl, S.; Zappa, F.; Mahr, I.; Lecointre, J.; Probst, M.; Mark, T. D.; Scheier, P. Phys. Rev. Lett. 2006, 97, 043201. 
(8) Bartelt, A.; Close, J. D.; Federmann, F.; Quaas, N.; Toennies, J. P. Phys. Rev. Lett. 1996, 77, 3525.

(9) Stienkemeier, F.; Higgins, J.; Ernst, W. E.; Scoles, G. Phys. Rev. Lett. 1995, 74, 3592 .

(10) Nauta, K.; Miller, R. E. Science 1999, 283, 1895.

(11) Nauta, K.; Miller, R. E. Science 2000, 287, 293.

(12) Diederich, T.; Döppner, T.; Braune, J.; Tiggesbäumker, J.; Meiwes-Broer, K.-H. Phys. Rev. Lett. 2001, 86, 4807.

(13) Müller, S.; Krapf, S.; Koslowski, T.; Mudrich, M.; Stienkemeier, F. Phys. Rev. Lett. 2009, 102, 183401.

(14) Vdovin, A.; Waluk, J.; Dick, B.; Slenczka, A. ChemPhysChem 2009, 10, 761.

(15) Spence, J. C. H.; Doak, R. B. Phys. Rev. Lett. 2004, 92, 198102.

(16) Hau-Riege, S. P.; London, R. A.; Chapman, H. N.; Szoke, A.; Timneanu, N. Phys. Rev. Lett. 2007, 98, 198302.

(17) Jurek, Z.; Faigel, G. Eur. Phys. J. D. 2008, 50, 35.

(18) von Haeften, K.; de Castro, A. R. B.; Joppien, M.; Moussavizadeh, L.; von Pietrowski, R.; Möller, T. Phys. Rev. Lett. 1997, 78, 4371.

(19) Fröchtenicht, R.; Henne, U.; Toennies, J. P.; Ding, A.; Fieber-Erdmann, M.; Drewello, T. J. Chem. Phys. 1996, 104, 2548.

(20) Peterka, D. S.; Lindinger, A.; Poisson, L.; Ahmed, M.; Neumark, D. M. Phys. Rev. Lett. 2003, 91, 043401. 
(21) Peterka, D. S.; Kim, J. H.; Wang, C. C.; Poisson, L.; Neumark, D. M. J. Phys. Chem. A 2007, 111, 7449.

(22) Buchenau, H.; Toennies, J. P.; Northby, J. A. J. Chem. Phys. 1991, 95, 8134.

(23) Wang, C. C.; Kornilov, O.; Gessner, O.; Kim, J. H.; Peterka, D. S.; Neumark, D. M. J. Phys. Chem. A 2008, 112, 9356.

(24) Loginov, E.; Rossi, D.; Drabbels, M. Phys. Rev. Lett. 2005, 95, 163401.

(25) Radcliffe, P.; Przystawik, A.; Diederich, T.; Döppner, T.; Tiggesbäumker, J.; Meiwes-Broer, K.-H. Phys. Rev. Lett. 2004, 92, 173403.

(26) Bonhommeau, D.; Lewerenz, M.; Halberstadt, N. J. Chem. Phys. 2008, $128,054302$.

(27) von Haeften, K.; Laarmann, T.; Wabnitz, H.; Möller, T. Phys. Rev. Lett. 2002, 88, 233401.

(28) Fárník, Michal; Samelin, B.; Toennies, J. P. J. Chem. Phys. 1999, 110, 9195.

(29) Fárník, M.; Henne, U.; Samelin, B.; Toennies, J. P. Phys. Rev. Lett. 1998, 81,3892 .

(30) Jiang, T.; Kim, C.; Northby, J. A. Phys. Rev. Lett. 1993, 71, 700.

(31) Nugent-Glandorf, L.; Scheer, M.; Samuels, D. A.; Mulhisen, A. M.; Grant, E. R.; Yang, X.; Bierbaum, V. M.; Leone, S. R. Phys. Rev. Lett. 2001, 87, 193002.

(32) Gagnon, E.; Ranitovic, P.; Tong, X.-M.; Cocke, C. L.; Murnane, M. M.; Kapteyn, H. C.; Sandhu, A. S. Science 2007, 317, 1374. 
(33) Antoine, P.; Foumouo, E.; Piraux, B.; Shimizu, T.; Hasegawa, H.;

Nabekawa, Y.; Midorikawa, K. Phys. Rev. A: At. Mol. Opt. Phys. 2008, 78, 023415.

(34) Pfeifer, T.; Spielmann, C.; Gerber, G. Rep. Prog. Phys. 2006, 69, 443.

(35) Haber, L. H.; Doughty, B.; Leone, S. R. Phys. Rev. A: At. Mol. Opt. Phys. 2009, 79, 031401.

(36) Peterka, D. S.; Kim, J. H.; Wang, C. C.; Neumark, D. M. J. Phys. Chem. B 2006, 110, 19945.

(37) Krause, J. L.; Schafer, K. J.; Kulander, K. C. Phys. Rev. Lett. 1992, 68, 3535.

(38) Corkum, P. B. Phys. Rev. Lett. 1993, 71, 1994.

(39) Salières, P.; L'Huillier, A.; Lewenstein, M. Phys. Rev. Lett. 1995, 74, 3776.

(40) Takahashi, E. J.; Nabekawa, Y.; Mashiko, H.; Hasegawa, H.; Suda, A.; Midorikawa, K. IEEE J. Sel. Top. Quant., 10, 1315.

(41) Henke, B. L.; Gullikson, E. M.; Davis, J. C. At. Data Nucl. Data Tables 1993, 54, 181.

(42) Kornilov, O.; Wilcox, R. B.; Gessner, O. in preparation.

(43) Eppink, A. T. J. B.; Parker, D. H. Rev. Sci. Instrum. 1997, 68, 3477.

(44) Johansson, A.; Raarup, M. K.; Li, Z. S.; Lokhnygin, V.; Descamps, D.; Lyngå, C.; Mevel, E.; Larsson, J.; Wahlström, C. G.; Aloise, S.; Gisselbrecht, M.; Meyer, M.; L'Huillier, A. Eur. Phys. J. D. 2003, 22, 3.

(45) Theodosiou, C. E. At. Data Nucl. Data Tables 1987, 36, 97. 
(46) Dribinski, V.; Ossadtchi, A.; Mandelshtam, V. A.; Reisler, H. Rev. Sci.

Instrum. 2002, 73, 2634.

(47) Joppien, M.; Karnbach, R.; Möller, T. Phys. Rev. Lett. 1993, 71, 2654.

(48) Ovchinnikov, M.; Grigorenko, B. L.; Janda, K. C.; Apkarian, V. A. J.

Chem. Phys. 1998, 108, 9351.

(49) Callicoatt, B. E.; Forde, K.; Ruchti, T.; Jung, L.; Janda, K. C.; Halberstadt, N. J. Chem. Phys. 1998, 108, 9371.

(50) Benderskii, A. V.; Zadoyan, R.; Schwentner, N.; Apkarian, V. A. J. Chem. Phys. 1999, 110, 1542.

(51) Benderskii, A. V.; Zadoyan, R.; Schwentner, N.; Apkarian, V. A. Proc. SPIE 1998, 3273, 297.

(52) Eloranta, J.; Apkarian, V. A. J. Chem. Phys. 2002, 117, 10139.

(53) Rosenblit, M.; Jortner, J. J. Phys. Chem. A 1997, 101, 751.

(54) Knuth, E. L.; Henne, U. J. Chem. Phys. 1999, 110, 2664.

(55) Jiang, T.; Northby, J. A. Phys. Rev. Lett. 1992, 68, 2620.

(56) Schulz, C. P.; Claas, P.; Stienkemeier, F. Phys. Rev. Lett. 2001, 87, 153401. 


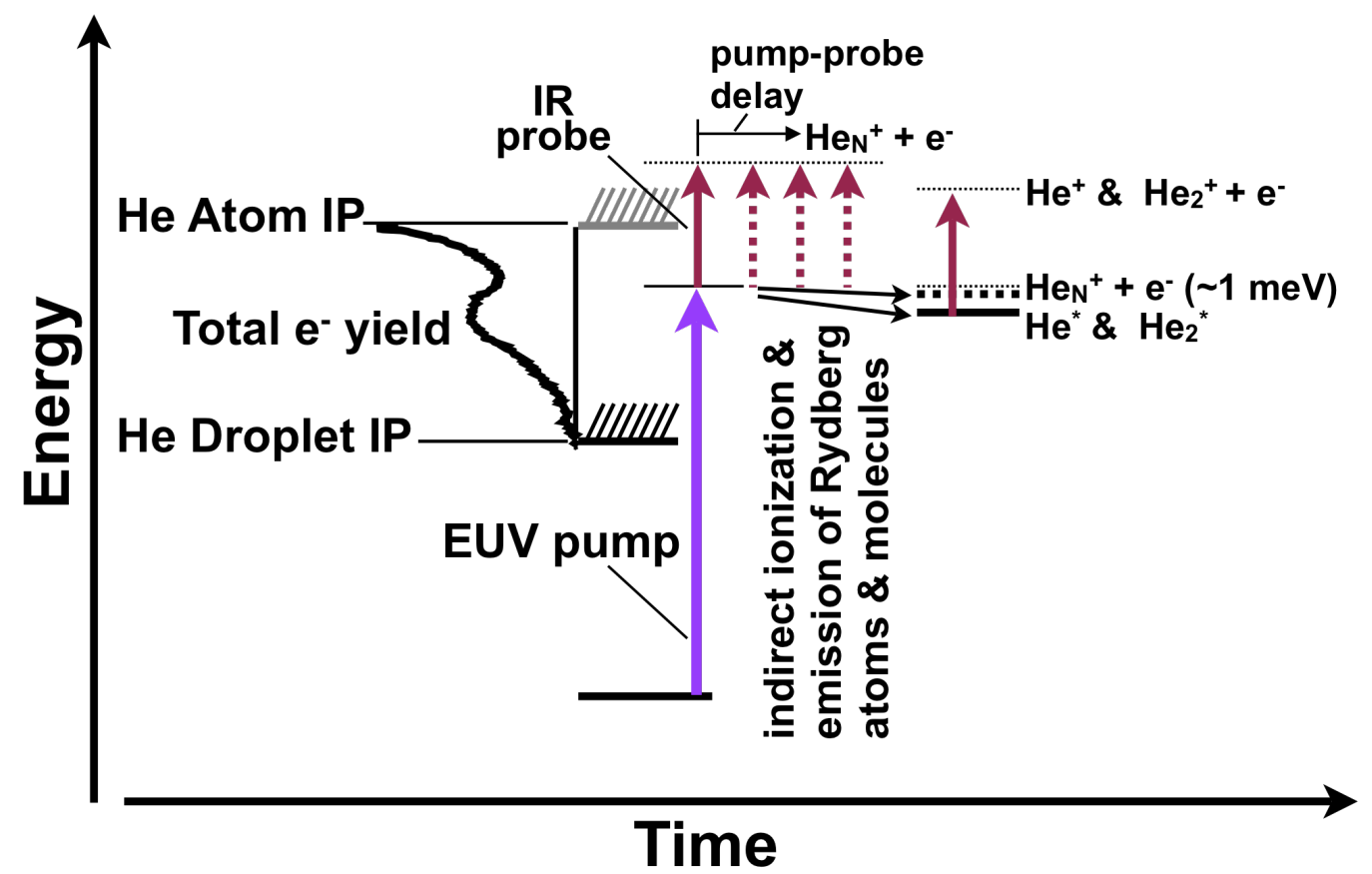

Figure 1. Femtosecond EUV pump - IR probe scheme to follow the relaxation dynamics of electronically excited helium droplets in real-time. 


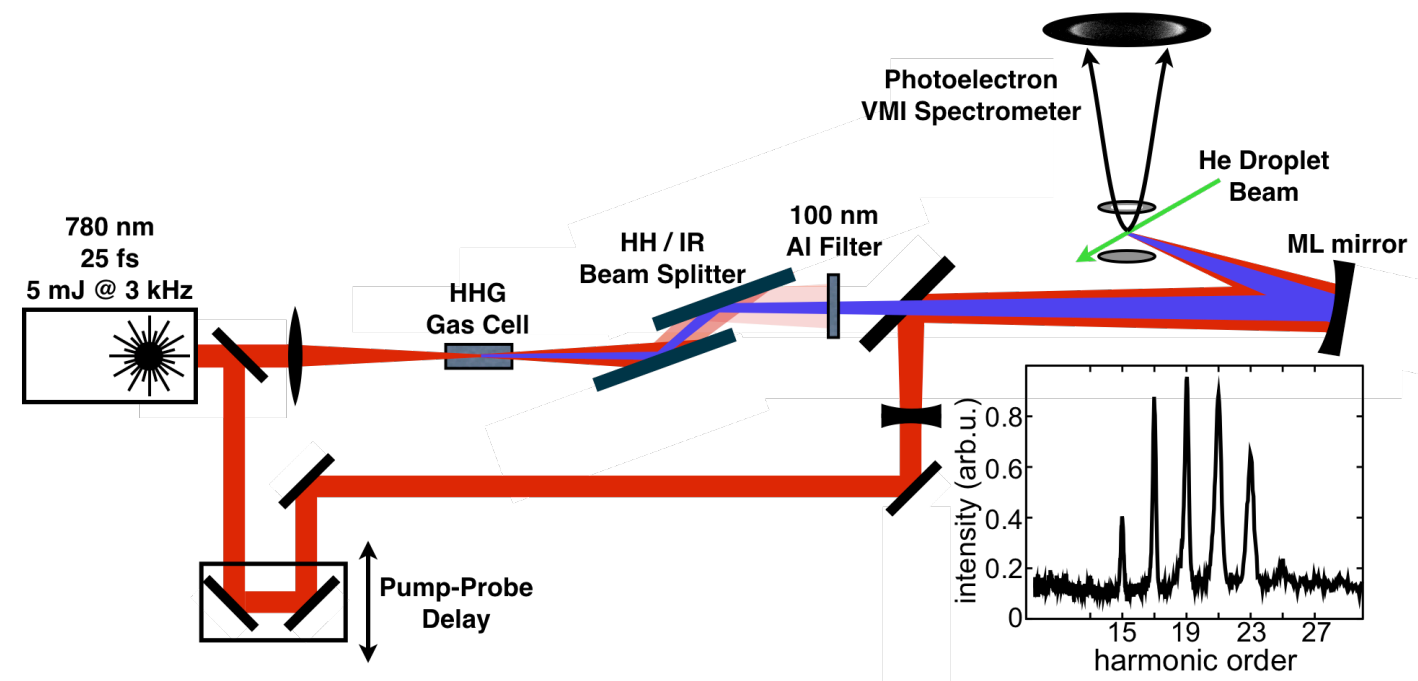

Figure 2. Setup to perform femtosecond time-resolved EUV photoelectron imaging experiments. The inset shows a high-order harmonic spectrum recorded in between the EUV-IR beam-combiner and the multilayer mirror. 


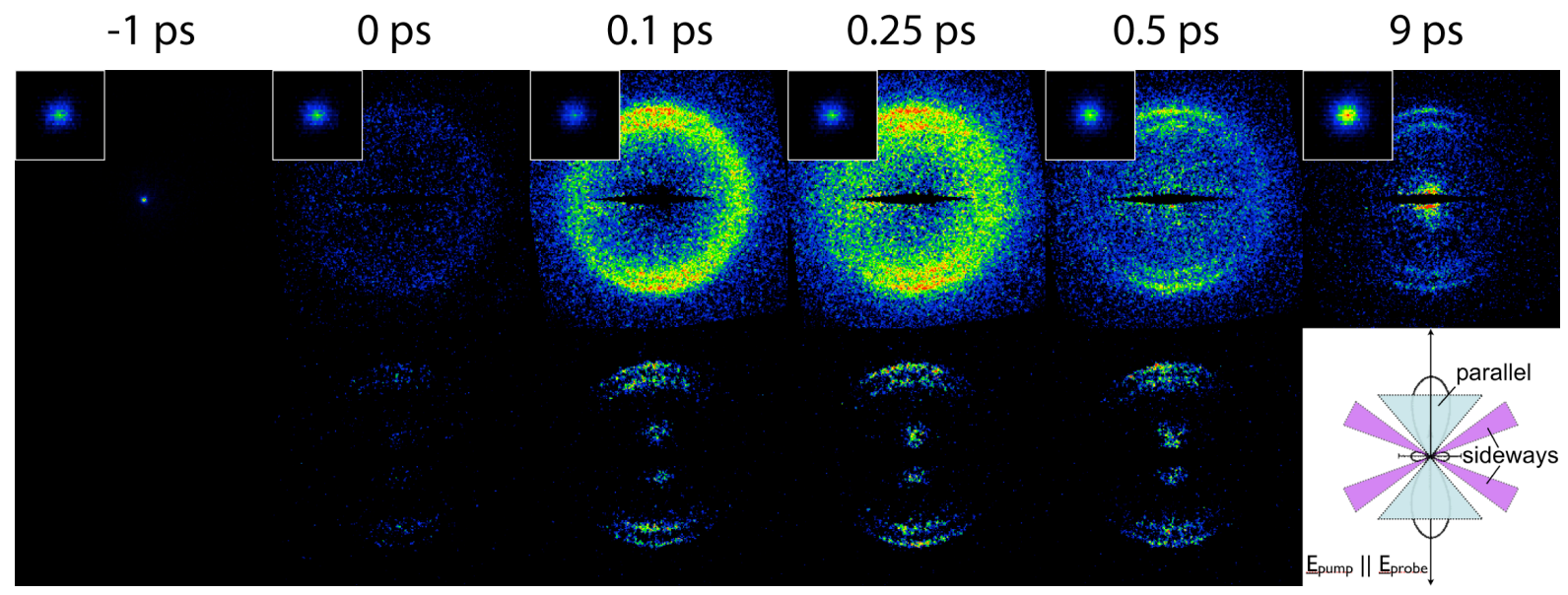

Figure 3. Femtosecond time-resolved photoelectron images of electronically excited helium droplets (upper row) and helium atoms. Pump and probe photon energies are centered at $23.7 \mathrm{eV}$ and $1.58 \mathrm{eV}$, respectively. Pump-probe time delays are noted above. Note that for time delays $\geq 0$ fs the sequence of images shows the difference between the "pump+probe" and "pump only" signals. The measurements for the fast photoelectrons in the outer regions of the detector and the center peak (insets) were performed separately to avoid saturation effects and MCP damage. The black areas in the center of the larger images are caused by a mask that covered the intense slow electron peak during the fast electron measurements. 


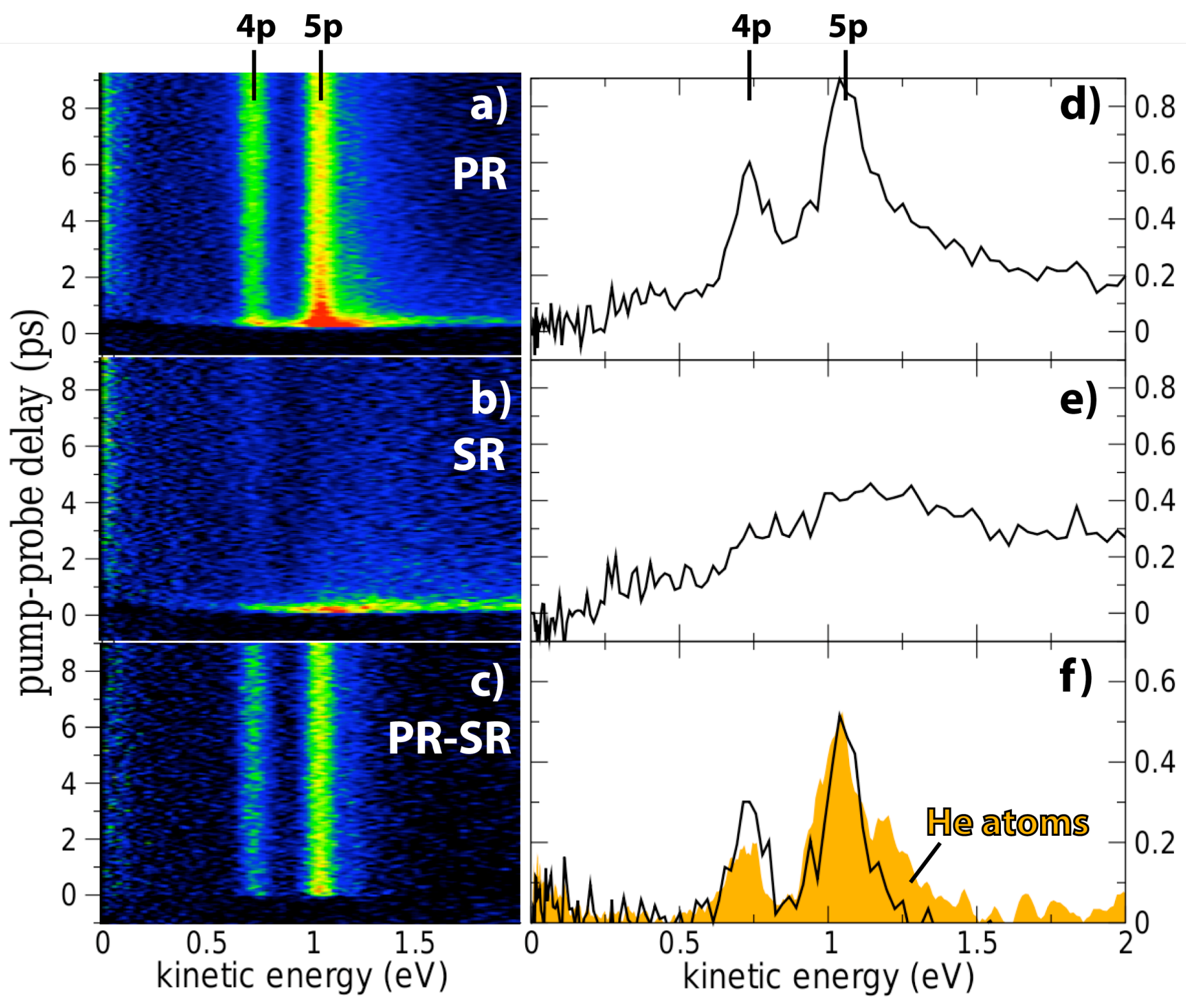

Figure 4. Left column: pump-probe time-delay-dependent photoelectron spectra: a) He droplet parallel emission, b) He droplet sideways emission, c) difference between signals in a) and b). Integration over delays $0 \mathrm{fs} \leq \Delta \mathrm{t} \leq 500 \mathrm{fs}$ in a), b), c) yields spectra d), e), f), respectively. The orange filled graph in f) shows a pumpprobe photoelectron spectrum of isolated He atoms. 


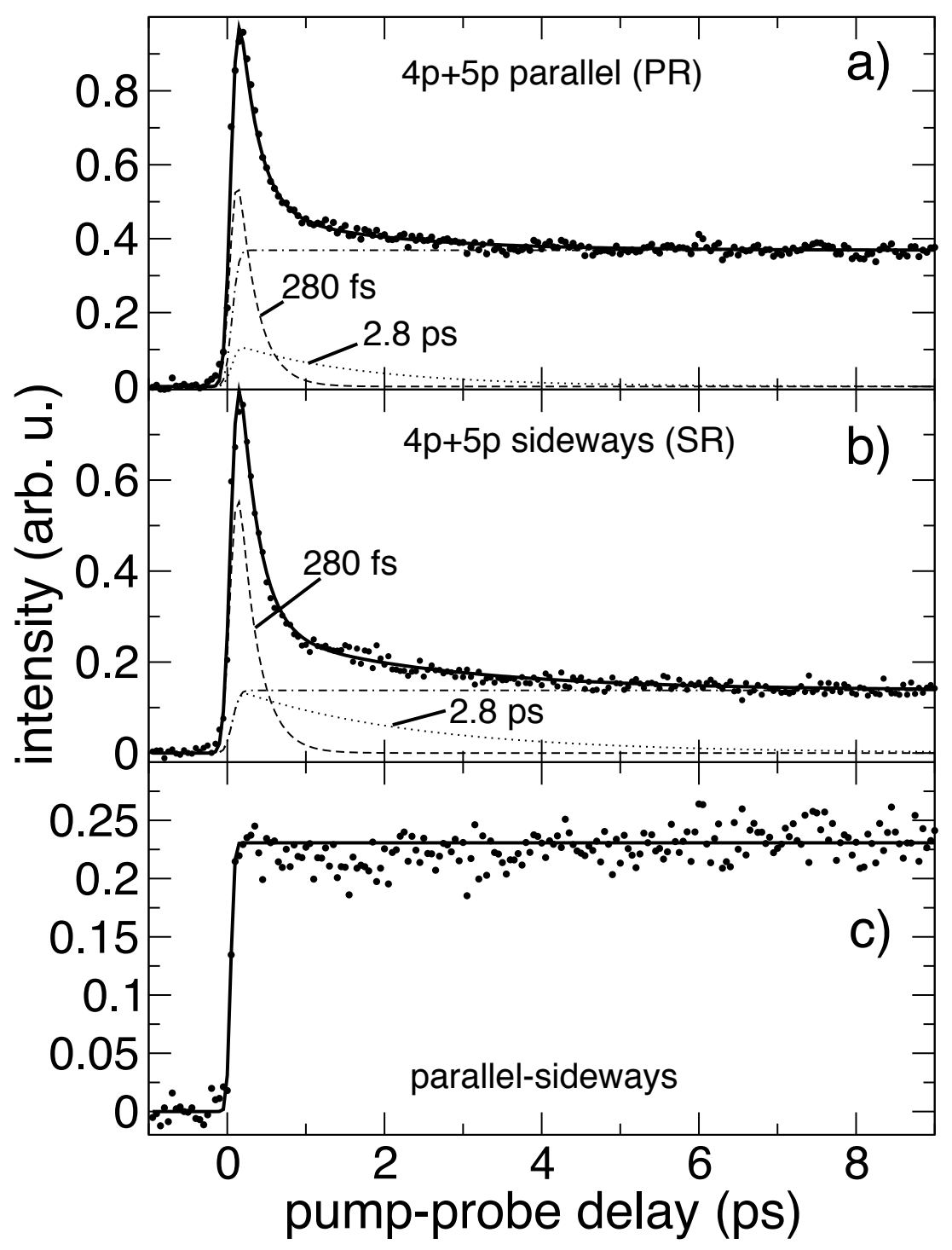

Figure 5. Transient intensities in the $n \mathrm{p}^{-1}(n=4,5)$ photoelectron peak regions; a) parallel emission, b) sideways emission, c) difference between a) and b) normalized to equal emission angle ranges. Solid curves are fits to model functions comprised of a fast decaying component, a slowly decaying component and a step function, convoluted with the apparatus function. Components are shown as dashed curves. 

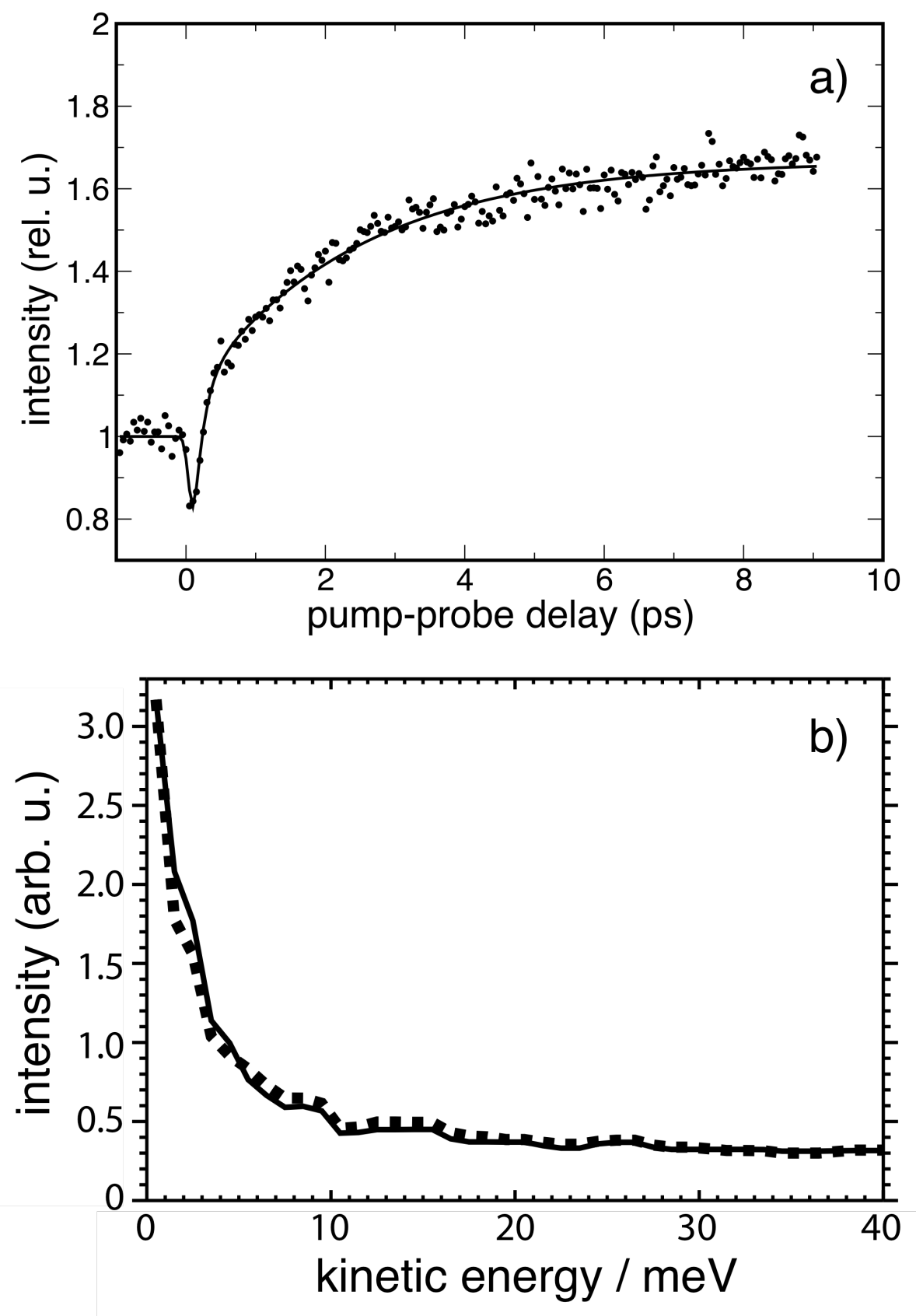

Figure 6. a) Transient intensity of the ultraslow photoelectron signal. The pump-probe signal is given relative to the signal induced by a single EUV pulse. b) Comparison of the ultraslow photoelectron peak measured by excitation with the pump pulse only (dotted) and the additional peak intensity that is induced by applying a probe pulse at $\sim 100$ ps pump-probe time delay (solid). 


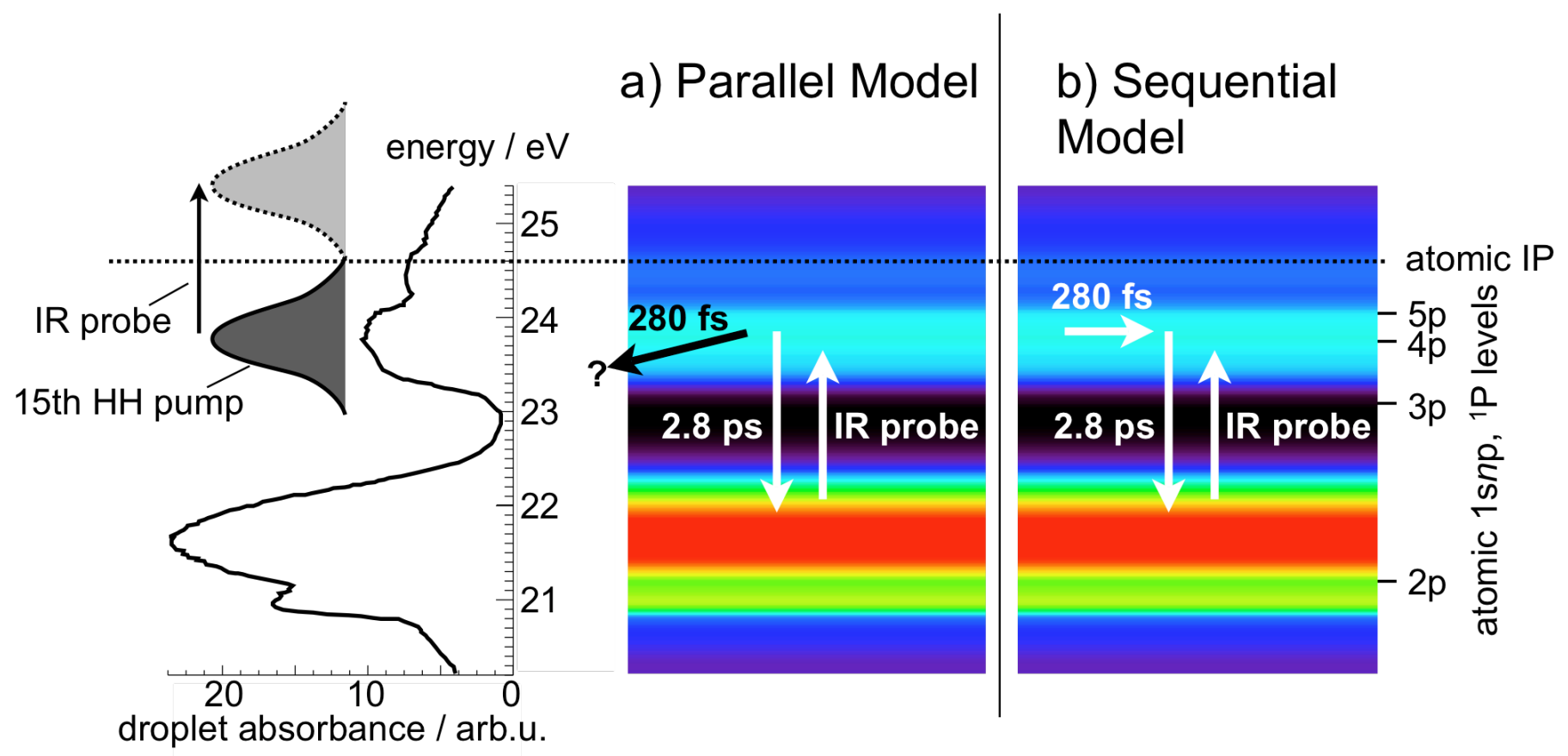

Figure 7. Proposed models for droplet relaxation mechanism. a) In the parallel model, two relaxation mechanisms with different time scales are initiated by the EUV excitation. b) In the sequential model, a fast intraband relaxation is followed by a slower interband relaxation. In both models, the droplet can be re-excited to the upper band by the IR probe pulse after relaxation to the lower electronic band. 